\title{
Formal Semantics for Property-Property Relations in SEAM Visual Language: Towards Simulation and Analysis of Visual Specifications
}

\author{
Irina Rychkova ${ }^{1}$ and Alain Wegmann ${ }^{1}$ \\ School of Communication and Computer Science, \\ École Polytechnique Fédérale de Lausanne (EPFL), \\ CH-1015 Lausanne, Switzerland. \\ \{rina.Rychkova, Alain.Wegmann\}eepfl.ch
}

\begin{abstract}
SEAM is an enterprise architecture method that defines a visual language for modeling. Our goal is to provide formal semantics for SEAM. Model simulation, model comparison, and refinement verification are practical benefits we expect from this formalization. This paper complements the existing SEAM semantics by formalizing property-property relations. This formalization is based on the theory of multi-relations and Relation Partition Algebra (RPA).
\end{abstract}

\section{Introduction}

In enterprise architecture projects, an enterprise, its environment, and its information systems are analyzed and designed. In general, the EA frameworks such as ISA [1], TOGAF [2] (for a more exhaustive list, see also [3]) do not propose a visual modeling notation. SEAM (Systemic Enterprise Architecture Methodology)[4] is a visual EA

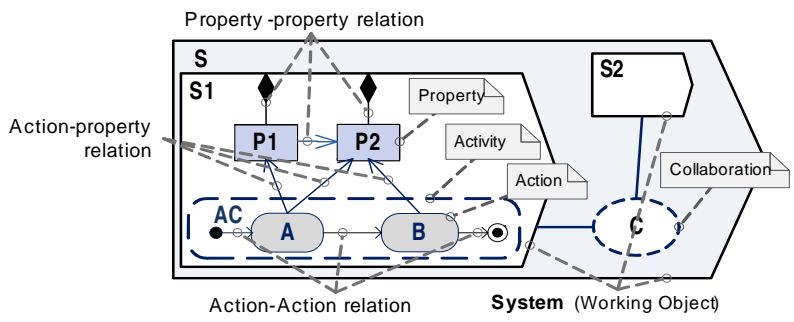

Fig. 1. SEAM visual notation.

method, based on Systems Thinking principles [5]. SEAM represents an enterprise and its environment as a hierarchy of systems (e.g. market, company, IT system, etc.)[4].

Figure 1 illustrates the SEAM visual notation: System $S$ is modeled as a collaboration of two systems $S 1$ and $S 2$. System $S 1$ is described by its observable properties $P 1, P 2$, and a behavior. The latter is represented by a set of actions $A, B$ organized within activity $A C$. SEAM specifies three types of relations between its elements: property-property relations, action-action relations, and action-property relations.

Our current research focuses on the definition of formal semantics for the SEAM visual language. In software engineering, formal methods have been successfully used in combination with UML[6] to formalize its visual notation, and to provide means for 
model analysis [7],[8]. However, to our knowledge, no such experience in the domain of EA is reported in literature. Model simulation [9], refinement verification [10], and model comparison for SEAM specifications are the main benefits we expect from this formalization.

In our previous work [10], formal semantics for SEAM properties, actions, activities, and action-property relations (Fig. 1) have been defined using higher-order logic and Refinement Calculus [11]. To complete the formalization of SEAM, the semantics for property-property relations and action-action relations has to be provided. This paper introduces a formal semantics for property-property relations, based on the Relation Partition Algebra (RPA)[13] and on the theory of multi-relations [12]. This semantics is especially useful for refinement propagation technique, explained in [10]: introduction, elimination, or modification of model elements (including property-property relations) affects the model correctness and consistency and requires model adjustments. Refinement propagation technique is based on the formal semantics of model elements. It defines the set of rules to enforce model consistency and correctness and allows to automate aforementioned adjustments.

This paper is organized as follows. In Section 2 we introduce the SEAM visual language and define its main modeling concepts. In Section 3 we present in more details the three types of relations defined in SEAM. In Section 4 we provide an extension of Relation Partition Algebra and the theory of multi-relations that formalizes the property-property relations in SEAM. Based on this formalization, we specify the consistency criteria for SEAM specifications. In Section 5 we discuss the related work. Section 6 presents our conclusions.

\section{The SEAM visual modeling language}

The SEAM ontology is based on the second part of the RM-ODP [14] specification. Based on this standard, the main modeling concepts such as property, state, action are defined. We briefly introduce these concepts below. For a detailed explanation, see [15].

Any system or system component in SEAM is modeled as a working object. We distinguish between the following views of a working object:

- Working object as a whole - a black box system specification;

- Working object as a composite - a white box system specification.

A working object as a whole ${ }^{1}$ describes a system by a number of properties $P_{1} \ldots P_{m}$ that specify data types, and behavior $\mathcal{B}$.

We distinguish between primitive and compound properties. The former can be considered as an alias for an operational data type (e.g. Int, String, Boolean, etc.); the latter is defined by a set of component properties and references to properties using propertyproperty relations.

A state of the primitive property denotes a value of the corresponding operational type (e.g. 1," $A B C$ ", true); a state of the compound property is defined by the states of its components and references.

\footnotetext{
${ }^{1}$ in this paper, we focus on modeling the working object as a whole and do not consider the working object as a composite, therefore the identifier 'as a whole' can be omitted
} 
A tuple of property instances and their corresponding values defines a system state $\sigma \in \Sigma$, where $\Sigma$ specifies a state space - a set of all possible states of the modeled system. A system state can be changed by a system behavior.

Behavior $\mathcal{B}$ of a working object can be seen as an action or as an activity.

Action $A$ is defined by a three-tuple $\{$ Pre, $U$, Post $\}$. Precondition Pre specifies a set of system states $\sigma \in \Sigma$ where $A$ is applicable. Postcondition Post specifies a set of system states $\sigma^{\prime} \in \Sigma$ after the application of $A$. $U$ specifies a state transition and is called update. Pre-, post- conditions, and updates are modeled as annotated action-property relations. Activity $A c$ can be considered as a detailed specification of action $A$ : it describes how the transition from pre- state to post- state is performed. Ac defines a set of component actions and the way they are composed to carry out the transition:

$$
A c \hat{=} A_{1} \bigcirc A_{2} \bigcirc \ldots \bigcirc A_{t}
$$

where $\bigcirc$ stands for component action ordering. This ordering is defined by actionaction relations.

\section{The Three Types of Relations in SEAM}

Action-Action(AA) Relations. SEAM specifies AA-relations using the BPMN [16] notation. Figure 2 illustrates activity $A C 1$ composed of four actions: $A, B, C$, and $D$.

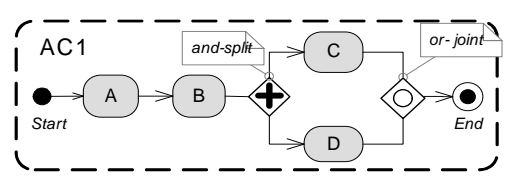

Fig. 2. Action-action relations.

An activity starts with a control action, called 'Start' and finishes with a control action 'End'. Actions $A$ and $B$ are connected by a transition relation that specifies a sequential invocation of $B$ after $A$ terminates. Action $B$ is connected with its successors by an and-split relation, which specifies that actions $C$ and $D$ are performed in parallel. Based on a joint-type (or-joint) of the last AA-relation towards the End symbol, the activity will terminate after at least one of the actions - $C$ or $D$ - terminates. We will address the formalization of AA-relations in our future work.

Action-Property(AP) Relations. Contrary to languages like UML [6], in which diagrams are specialized (e.g. class diagram, state diagram, activity diagram), SEAM describes system behavior and data structure within one diagram and provides explicit relations between them (Fig. 3). A group of expressions on the destination end specifies an information, useful for specification simulation. Target expressions specify the relation type: Pre-, Post- conditions, or Updates; instance expressions specify the instance names to be used by the corresponding target expressions; select expressions (optional) specify the instance choice providing multiple instances available. In UML, this information is usually provided by annotated OCL[22] expressions.

Action GDiv in Fig. 3 specifies a division operation and selects the greatest devisor if more then one is available. 


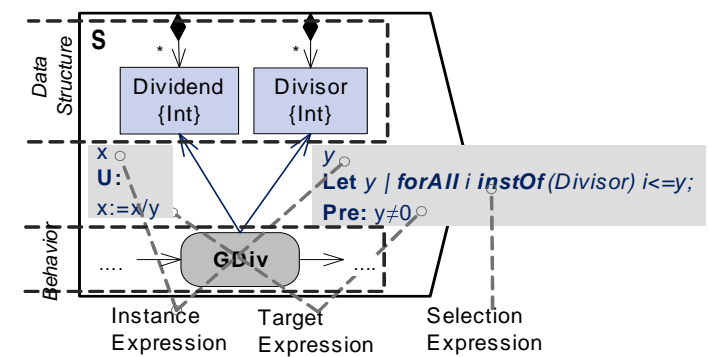

Fig. 3. Action-property relations annotated with instance, selection, and target expressions.

Property-Property (PP) Relations. Relation Partition Algebra (RPA) [13] defines 'partof' and 'use' relations as a special type of binary relations. These terms can be used to represent PP-relations in SEAM. Consider a system data structure defined by a number of data types (properties). The modularization of the data types (definition of compound properties) gives rise to the part-of relations (Fig. 4-b, 5-a). Mechanism, when one property references (uses) another, can be modeled by use relations (Fig. 4-c)Fig. 5-b).

In SEAM, part-of relations are used to designate the context, in which a property exists. These relations are depicted by a line with a 'black diamond' at its destination end and an expression at its source end, to be read "[source] is a part of [destination]". Use relations are depicted by a line with an expression at its destination end, to be read [source] references (uses) [destination]. Expressions specify relation multiplicity (usually, an integer-valued interval with a possibly infinite upper bound) and a list of instance names. The multiplicity constrains the minimum and the maximum allowable number of instances of a given property in the system. The multiplicity of the opposite relation end is constant and equal to 1 in the SEAM specifications (usually omitted).

\section{Formalization of Property-Property Relations Using RPA}

The Relation Partition Algebra (RPA) by Feijs and van Ommering [13] defines partof and use relations as special types of binary relations. The theory of multi-relations by Feijs and Krikhaar [12] defines a formalism, suitable for reasoning about relation multiplicities. We combine these theories and formalize PP-relations in SEAM as partof and use relations with multiplicities that can be also called SEAM multi-relations.

\subsection{SEAM Multi-Relations}

Multi-relation $m(x, y)=n$ (Fig. 4-a), defined in [12], specifies $n$ occurrences of the binary relation $(x, y)$. Where $x \in X, y \in Y$ - are elements of corresponding sets.

SEAM multi-relations part and use (Fig. 4-b,c) between properties $P$ and $Q$, and $P$ and $T$, where $P, Q, T \in \mathbb{P}$, specify 'relations with multiplicities' between instances $x: P, y: Q, z: T$ of corresponding properties.

SEAM multi-relations part and use on $\mathbb{P}$ are defined by pairs of total functions:

$$
\begin{gathered}
\text { part }_{\text {inf }}, \text { use }_{\text {inf }}: \mathbb{P} \times \mathbb{P} \rightarrow \mathbb{N} \cup\{\infty\}, \quad \text { part }_{\text {sup }}, \text { use }_{\text {sup }}: \mathbb{P} \times \mathbb{P} \rightarrow \mathbb{N} \cup\{\infty\} \\
0 \leq \text { part }_{\text {inf }} \leq \text { part }_{\text {sup }} \leq \infty, \quad 0 \leq \text { use }_{\text {inf }} \leq \text { use }_{\text {sup }} \leq \infty
\end{gathered}
$$

There are at least $n_{1}$ and at most $n_{2}$ instances of property $P$ for each instance of $Q$ : 
(a)

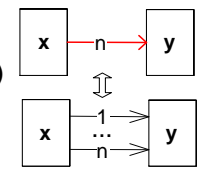

$m(x, y)=n$ (b)

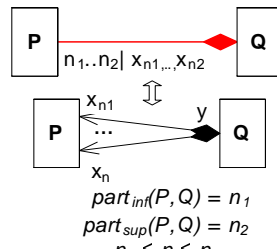

(c)

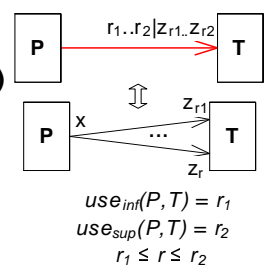

Fig. 4. SEAM multi-relations. a) binary multi-relation; b) SEAM 'part-of' relation: ' $P$ is a part of $Q$ '; c) SEAM 'use' relation: ' $P$ uses $T$ '. Above: short notation, below: detailed notation.

$$
\operatorname{part}_{\mathrm{inf}}(P, Q)=n_{1}, \operatorname{part}_{\mathrm{sup}}(P, Q)=n_{2} \Leftrightarrow \forall y: Q \exists x_{1}, . ., x_{n}: P \mid n_{1} \leq n \leq n_{2}
$$

There are at least $r_{1}$ and at most $r_{2}$ instances of property $T$ used (or referenced) by each instance of property $P$ :

$$
\text { use }_{\text {inf }}(P, T)=r_{1}, \text { use }_{\text {sup }}(P, T)=r_{2} \Leftrightarrow \forall x: P \exists z_{1}, . ., z_{r}: T \mid r_{1} \leq r \leq r_{2}
$$

Above, $n$ and $r$ are the corresponding actual number of instances.

Example 1. Figure 5-a illustrates the part-of relation between properties $P$ and $Q$ : part $_{\text {sup }}(P, Q)=M_{1}$; $\operatorname{partinf}_{\text {inf }}(P, Q)=0$, where ' $P$ is a part of $Q$ ' and there exist at most $M_{1}$ instances of $P$ for each instance of $Q . x_{1}, . ., x_{M_{1}}$ - is a list of available instance names.

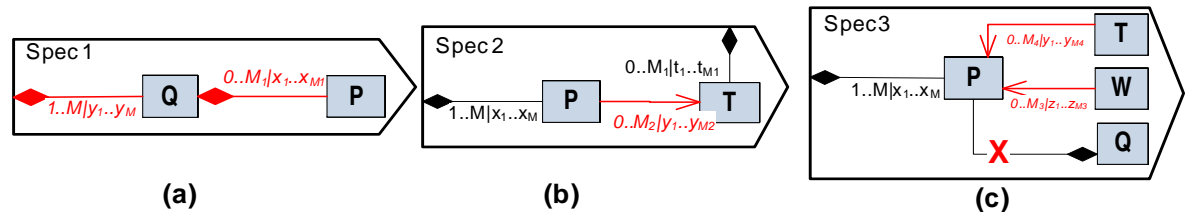

(a)

(b)

(c)

Fig. 5. Property-property relations annotated with multiplicity and instance expressions. a) partof relations; b) use relation; c)Well-formedness of PP-relations.

Example 2. Figure 5-b illustrates the use relation between properties $P$ and $T$ : $\operatorname{part}_{\text {sup }}(P, T)=M_{2}$; $\operatorname{part}_{\text {inf }}(P, T)=0$, where ' $P$ references (uses) $Q$ ' and there exist at most $M_{2}$ references on $T$ for each instance of $P . y_{1}, . ., y_{M_{2}}$ - is a list of available reference names.

Similarly to [13], we define a relation composition $\circ$ of SEAM multi-relations (Fig. 6:

$$
s m_{1} \circ \operatorname{sm}_{2}=\left\{(P, R) \mid \exists Q \in \mathbb{P} \bullet \operatorname{sm}_{1}(P, Q) \wedge \operatorname{sm}_{2}(Q, R)\right\}
$$

Identity relation $I$ is a neutral element: $I \circ s m=s m \circ I=s m$.

For properties $P, Q, R \in \mathbb{P}$ we write:

$$
\begin{gathered}
\left(s m_{1} \circ s m_{2}\right)_{\text {inf }}(P, R)=\sum_{Q \in \mathbb{P}} s m_{1 \text { inf }}(P, Q) \cdot s m_{2 \inf }(Q, R) \\
\left(s m_{1} \circ s m_{2}\right)_{\text {sup }}(P, R)=\sum_{Q \in \mathbb{P}} s m_{1 \text { sup }}(P, Q) \cdot s m_{2 \sup }(Q, R) \\
I_{\text {sup }}(P, Q)=I_{\text {inf }}(P, Q)=0 \text { if } P \neq Q
\end{gathered}
$$

where the following holds: 


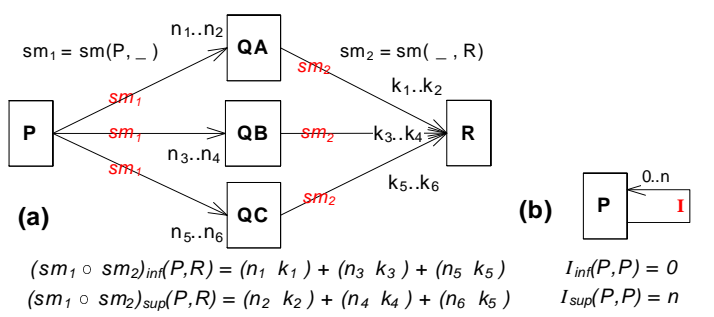

Fig. 6. a) SEAM multi-relation composition. $s m_{1}$ - a relation with source $P, s m_{2}$ - a relation with destination $R$; b) Identity relation.

$$
\left(s m_{1} \circ s m_{2}\right) \circ s m_{3}=s m_{1} \circ\left(s m_{2} \circ s m_{3}\right)=s m_{1} \circ s m_{2} \circ s m_{3}
$$

We define the exponentiation for SEAM multi-relations on $\mathbb{P}$ as $s m^{n}=s m \circ s m . . \circ s m$ (n-times), putting $s m^{0}=I$ - identity relation.

We define a transitive closure $\mathrm{sm}^{+}\left(P_{1}, P_{2}\right)$ on $\mathbb{P}$ iff there exists a sequence of elements $Q_{i} \in \mathbb{P}, i=1$..n such that $P_{1}=Q_{1}$ and

$$
\begin{gathered}
\operatorname{sm}\left(Q_{1}, Q_{2}\right) \circ \ldots \circ \operatorname{sm}\left(Q_{n-1}, Q_{n}\right) \circ \operatorname{sm}\left(Q_{n}, P_{2}\right)=\operatorname{sm}^{n}\left(P_{1}, P_{2}\right) . \\
s m_{\text {inf }}^{+}=\bigcup_{n=1}^{\infty} s m_{\text {inf }}^{n} \text { and } s m_{\text {sup }}^{+}=\bigcup_{n=1}^{\infty} s m_{\text {sup }}^{n}
\end{gathered}
$$

Here $\operatorname{sm}^{n}\left(P_{1}, P_{2}\right)$ is an n-step path from $P_{1}$ to $P_{2}$.

\section{2 'Part-Of'}

A part-of relation between properties $P$ and $Q$ (Fig. 4-b) specifies the fact that property $P$ is a part of a data type, defined by property $Q$ (see Example 1$)$. Property $P$ can be a part of one and only one compound property, i.e. part-of relations is functional:

$$
\forall P, Q, R \in \mathbb{P}_{p^{\prime}} \operatorname{rrt}_{\mathrm{sup}}(P, Q)>0 \wedge \operatorname{part}_{\mathrm{sup}}(P, R)>0 \Leftrightarrow Q=R
$$

Property $P$ cannot be a part of itself, and there is no path of one or more legs that starts at $P$ and leads back to $P$, i.e. part-of relations is cycle-free, as defined in [13]:

$$
\forall P \in \mathbb{P} \quad \operatorname{part}^{+}(P, P)=\emptyset
$$

We define a part-of relation between a property and a system (a working object):

$$
\text { part }_{\text {sup }}: \mathbb{P} \rightarrow \mathbb{N} \cup\{\infty\}, \quad \text { part }_{\text {inf }}: \mathbb{P} \rightarrow \mathbb{N} \cup\{\infty\}
$$

Here, part-of relation specifies a collection of instances of $P$ provided by the system. These instances can be identified with the global variables of the system.

Example 3. Figure 5-a illustrates the part-of relation where ' $Q$ is a part of Spec 1 ' and there exist at most $M$ instances of $Q$ in $\operatorname{Spec}_{1}: \operatorname{part}_{\mathrm{sup}}(Q)=M ; \operatorname{part}_{\mathrm{inf}}(Q)=1$, and $y_{1}, \ldots, y_{M}$ - is a list of available instance names.

Dynamic creation and deletion of property instances is an important issue that can be specified on the diagram, using part-of relations, prior to model simulation. 
Example 4. In Fig.5-a, consider some action Create $Q$ that creates instances of property $Q$, action Delete $Q$ that deletes them, and $M_{a c t}$ - the actual number of instances of $Q$ in the system (i.e. a number of $y_{i}$ at a given moment of simulation), where $1 \leq M_{a c t} \leq M$.

Using multiplicities, the effect of creation of an instance $y_{i}$ can be expressed by the following statement: $M_{a c t}:=M_{a c t}+1$. For instance deletion we can write: $M_{a c t}:=M_{a c t}-1$. This can be interpreted as follows:

1)If $M \in \mathbb{N}$ is a constant - every time, after a new instance of $Q$ is dynamically created, one more name $y_{i}$ is taken from the list of available instance names $y_{1} . . y_{M}$. When $M_{a c t}=M-$ Ceate $Q$ must not be available any more.

2)If $M=\infty$ - Create $Q$ is not restricted.

3) Every time one instance of $Q$ is deleted, one name $y_{i}$ is put back to the list $y_{1} . . y_{M}$. When $M_{a c t}=1$ - Delete $Q$ must not be available any more.

We generalize the assertion that part-of relation is functional eq.(6) for the part-of relation compositions:

Lemma 1. For each ordered pair of properties $\left\langle P_{1}, P_{2}\right\rangle$ there exists at most 1 sequence of properties $Q_{1}, . ., Q_{n} \in \mathbb{P}$ with $P_{1}=Q_{1}$ and a corresponding sequence of partof relations

$$
\operatorname{part}\left(Q_{1}, Q_{2}\right) \circ \ldots \circ \operatorname{part}\left(Q_{n-1}, Q_{n}\right) \circ \operatorname{part}\left(Q_{n}, P_{2}\right)=\operatorname{part}^{n}\left(P_{1}, P_{2}\right)
$$

such that $\operatorname{part}^{n}\left(P_{1}, P_{2}\right) \equiv \operatorname{part}^{+}\left(P_{1}, P_{2}\right)$.

Here $\operatorname{part}^{n}\left(P_{1}, P_{2}\right)$ is a path from $P_{1}$ to $P_{2}$ of the length $n$, where ' $P_{1}$ is a part of $Q_{2}$, and $Q_{2}$ is a part of $Q_{3}$, and .. and $Q_{n}$ is a part of $P_{2}$ '.

This lemma stipulates that between two properties can be found at most one sequence of 'part-of' relations of an arbitrary length and this sequence is linear.

We can generalize the definition of part-of relations for relation composition:

Definition 1. Iffor two properties $P$ and $Q$ there exists some $n>0$ such that $\operatorname{part}^{n}(P, Q) \neq$ $\emptyset$ then $Q$ contains $P$ as a part.

Corollary 1. For each property $P$ there exists at most one property $Q \in \mathbb{P}$ such that $Q$ contains $P$ as a part, and part ${ }^{n_{\max }}(P, Q)=\operatorname{part}^{+}(P, Q) \neq \emptyset$ Here $n_{\text {max }}-$ is the longest path that starts at $P$ and finishes at $Q$.

By Lemma 1 and Corollary 1 we can calculate the maximum and minimum number of instances of property $P$ in the system $S$ :

$$
\begin{gathered}
\operatorname{Inst}_{\text {max }}(P)=\text { part }_{\mathrm{sup}}(P)+\sum_{Q \in \mathbb{P}} \operatorname{part}_{\mathrm{sup}}^{+}(P, Q) \cdot \operatorname{part}_{\mathrm{sup}}(Q) \\
\operatorname{Inst}_{\text {min }}(P)=\operatorname{part}_{\mathrm{inf}}(P)+\sum_{Q \in \mathbb{P}} \operatorname{part}_{\mathrm{inf}}^{+}(P, Q) \cdot \operatorname{part}_{\mathrm{inf}}(Q)
\end{gathered}
$$

\section{3 'Use'}

A use relation between properties $P$ and $T$ (Fig. 4 -c) specifies the fact that property $P$ references property $T$ (see Example 2). Property $T$ can be referenced by multiple compound properties, i.e. use relations is non-functional:

$\exists T, P, P^{\prime} \in \mathbb{P} \mid P \neq P^{\prime} \bullet$ use $_{\text {sup }}(P, T)>0 \wedge$ use $_{\text {sup }}\left(P^{\prime}, T\right)>0$ 
Property $T$ can be referenced by itself, i.e. use relations can be cyclic as defined in [13]:

$$
\exists T \in \mathbb{P} \mid \quad \operatorname{use}^{+}(T, T) \neq \emptyset
$$

We can calculate the number of references to $T$ in the system:

$$
\forall P_{i} \mid \text { use }_{\text {sup }}\left(P_{i}, T\right)>0, \quad \operatorname{Ref}\left(P_{i}, T\right)=\operatorname{Inst}_{\text {max }}\left(P_{i}\right) \cdot \text { use }_{\text {sup }}\left(P_{i}, T\right)
$$

and the maximum number of references:

$$
\operatorname{Ref}_{\max }(T)=\max _{P_{i}}\left(\operatorname{Inst}_{\max }\left(P_{i}\right) \cdot \text { use }_{\text {sup }}\left(P_{i}, T\right)\right)
$$

\subsection{From Property-Property Relations to Specification Well-Formedness and Consistency}

PP-relations define a data structure in SEAM specifications. To obtain the well-formed data structure, the following must be ensured for each system property $P$ :

-Property $P$ can be a part of one and only one compound property or a working object; -Property $P$ cannot be a part of itself, and there is no path of two or more legs that starts at $P$ and leads back to $P$.

Formalization of PP-relations enables us to detect errors concerned with data structure inconsistency. For example, an instantiation deficiency, when for some property $P$ the number of declared instances in the specification $\operatorname{Inst}_{\max }(P)$ is less then required by the system (specified by references from other properties):

$$
\exists Q \in \mathbb{P} \mid \operatorname{Re} f_{\text {max }}(Q, P)>\text { Inst }_{\text {max }}(P)
$$

Example 5. In Fig. 5-c, property $P$ is referenced by two properties $T$ and $W$. To avoid instantiation deficiency, the specification must guarantee that $\max \left\{M_{4}, M_{3}\right\} \leq M$, i.e. the number of references on $P$ from either $W$ or $T$ must not exceed the number of instances $M$ of $P$, defined by the specification.

A free-floating property is a data type that is not instantiated in the system.

Definition 2. Property $P$ is free-floating iff $\operatorname{Inst}_{\max }(P)=0$.

Example 6. In Fig. 5-c, properties $T, W, Q$ are free-floating:

$$
\operatorname{Inst}_{\text {max }}(T)=\operatorname{Inst}_{\text {max }}(W)=\operatorname{Inst}_{\text {max }}(Q)=0 .
$$

A property can be specified as a free-floating, when the number of instances is not important at a given level of abstraction. For example, a number of 'items for sale' might be omitted in an abstract specification of a vending machine, since a behavior of this machine is the same for each item.

If free-floating property $P$ is referenced by some property $Q$, i.e.

$$
\exists Q \in \mathbb{P} \mid \operatorname{Re} f_{\text {max }}(Q, P)>0
$$

this causes an instantiation deficiency in the specification: $\operatorname{Re}_{\max }(Q, P)>0=\operatorname{Inst}_{\text {max }}(P)$. We summarize with the following criteria of consistency:

System data structure defined by SEAM specification is consistent if:

1. all part-of relations in the specification are functional and cycle-free (i.e. eq. (6) and eq. (7) hold),

2. instance declaration is sufficient:

$$
\forall P \in \mathbb{P} \quad \operatorname{Ref}_{\text {max }}(P) \leq \operatorname{Inst}_{\text {max }}(P)
$$

3. no reference on a free-floating property exists:

$$
\forall P \in \mathbb{P} \mid \text { Inst }_{\text {max }}(P)=0 \quad \Rightarrow \quad \text { use }\left(P_{i}, P\right)=\emptyset \forall P_{i} \in \mathbb{P}
$$




\section{Related work}

The scientific publications, listed below, report some practical applications in the area of visual model analysis based on a combination of visual and formal methods:

Pons [8] presents the OCL-based technique and a tool support for UML and OCL model refinement. Object- $Z$ is an underlying theory for refinement verification. The authors discuss the refinement patterns and formulate the refinement conditions for these patterns in OCL [22].

Muskens et al. [23] focuse on the problem of consistency checking between software views, expressed as UML diagrams. The approach in [23] is based on verification of obligations and constraint rules using relation partition algebra.

Modeling languages, listed below, consider formalization of their visual notation as a bridge to model simulation.

OPM (Object-Process Methodology)[17] proposes a method for the complete integration of the system' states and behaviors within a single graphical model. OPM distinguishes different types of relations between its model elements, similarly to SEAM. An Object-Process Language (OPL) serves as a basis for generation an executable code and a database schema and represents a formal semantics for OPM.

DEMO (Design \& Engineering Methodology for Organizations) [18] is a method for (re)designing organizations. DEMO provides a semantics for model simulation.

BPMN (Business Process Modeling Notation)[16] provides a visual notation for business process modeling (BPM). SEAM action-action relations are defined based on BPMN. Explicit bindings between a BPMN process and the data this process operates on (represented by action-property relations in SEAM) is made by annotation with BPEL constraints. BPEL (Business Process Executable Language)[19] was developed for business process model simulation and verification. An automated mapping of BPMN diagrams to BPEL for further execution is supported by many commercial tools (e.g. iGrafx, IBM WBI Modeler, etc - see http://www.bpmn.org/BPMN_Supporters.htm\#current for an exhaustive list). Formal semantics for business process modeling was provided using Petri Net [20].

UML (Unified Modeling Language) [21], [6] defines a set of specialized diagrams for its models. System behavior is addressed in UML by activity diagrams. The data structure of a system is captured by UML class diagrams. A class diagram defines classes, organized within a model using association, aggregation, composition, and inheritance relations. Part-of relation in SEAM can be identified with UML composition, whereas SEAM use relation is defined by analogy with UML association. The semantics of activity diagrams in UML 2.0 is based on Petri Nets[7]. However, there were many attempts to define this semantics based on other formal languages: LOTOS, ASM, CSP, LTS (see [7] for details).

\section{Conclusion}

In this work, we introduce the formal semantics of property-property (PP) relations in SEAM visual language. This formalization enables us reasoning about consistency of data structure.

In our previous work [10], the refinement propagation technique for SEAM visual specifications has been introduced. The proposed algorithm explores the possible con- 
flicts between model elements, caused by refinement, and applies specific rules of refinement propagation to enforce the model correctness.

Model refinement where property-property relations are eliminated, introduced, or modified is not considered in [10] and can be captured using the theory proposed in this work. Refinement propagation rules that enforce model consistency and wellformedness, as defined at the end of section 4, can be introduced. For instance, such a rule may forbid the user from deleting a part-of relation because it will lead to an instantiation deficiency. Alternatively, an alert can be generated in the case of a creation of a part-of relation if it leads to a cycle. Automated model refinement is the main practical benefit expected from the proposed formal semantics.

\section{References}

1. Zachman J. A.: A Framework for Information Systems Architecture, IBM Systems Journal (1987)

2. The Open Group Architecture Framework (TOGAF), The Open Group,(2006)

3. Schekkerman, J.: How to Survive in the Jungle of Enterprise Architecture Frameworks: Creating or Choosing an Enterprise Architecture Framework, Trafford, (2003).

4. Wegmann, A.: On the systemic enterprise architecture methodology (SEAM). In proceedings of International Conference on Enterprise Information Systems (ICEIS) (2003)

5. Weinberg, G.M.: An Introduction to General Systems Thinking. Wisley \& Sons (1975)

6. Unified Modeling Language (UML), v. 2.1.1. OMG (2007)

7. Störrle, H.: Semantics of UML 2.0 Activities, Proceedings of the IEEE Symposium on Visual Languages and Human-Centric Computing (VL/HCC) (2004).

8. Pons, C.: Heuristics on the definition of UML refinement patterns. SOFSEM, (2006).

9. Rychkova, I., Wegmann, A.: A Method for Functional Alignment Verification in Hierarchical Enterprise models. BUSITAL workshop in CAiSE (2006)

10. Rychkova, I., Wegmann, A.: Refinement propagation. Towards automated construction of visual specifications. International Conference on Enterprise Information Systems (ICEIS) (2007)

11. Back, R.-J., von Wright, J.: Refinement Calculus: A Systematic Introduction. Springer (1998)

12. Feijs, L.M.G., Krikhaar R.L.: Relation algebra with multi-relations. International Journal of Computer Mathematics.(1998)

13. Feijs, L.M.G., van Ommering, R.C: Relation partition algebra - mathematical aspects of uses and part-of relations. Science of Computer Programming (1999)

14. Reference model of open distributed processing. Draft International Standard (DIS)(1995)

15. Wegmann, A., Naumenko, A.: Conceptual Modeling of Complex Systems Using an RMODP Based Ontology. 5-th IEEE International Enterprise Distributed Object Computing Conference (EDOC) (2001)

16. BPMN 1.0: Final Adopted Specification. OMG. (2006)

17. Dori, D., Object-Process Methodology, A Holistic Systems Paradigm. Springer (2002).

18. Dietz, J. L. G.: Enterprise Ontology Theory and Methodology. Springer (2006)

19. Business Process Execution Language for Web Services Version 1.1. The IBM. (2004)

20. van der Aalst, W.: Challenges in business process management: Verification of business processes using petri nets. Bulletin of the EATCS.(2003)

21. Rumbaugh, J., Jacobson, I., and Booch, G.: The Unified Modeling Language Reference Manual, Second Edition. Addison-Wesley. (2005)

22. OCL 2.0 Final Adopted Specification. OMG (2003)

23. Muskens, J., Bril, R.J., Chaudron, M.R.V.: Generalizing consistency checking between software views. Conference on Software Architecture (WICSA), IEEE (2005) 